\title{
Arbeitsmarktreformen in Deutschland - Zwischen Pfadabhängigkeit und Paradigmenwechsel
}

\author{
Frank Oschmiansky \\ Andreas Mauer \\ Karin Schulze Buschoff
}

Die Reformen der letzten Jahre bewirkten die größten Akzentverschiebungen in der Arbeitsmarktpolitik seit der Einführung des Arbeitsförderungsgesetzes (AFG) im Jahr 1969. Im Bereich der aktiven Arbeitsmarktpolitik sind sowohl in der Logik verbleibende pfadabhängige Krisenbewältigungsstrategien als auch Veränderungen der Zielsetzungen zu verzeichnen. Neu ist die hohe Dynamik, mit der Reformen vorangetrieben werden. Die Vielzahl der gesetzlichen Änderungen und die damit einhergehende Entwicklung in der Vermittlungs- und Förderungspraxis haben in der Summe zu einem Paradigmenwechsel weg von der aktiven hin zu einer autoritär aktivierenden Arbeitsmarktpolitik geführt. Die Förderung des Niedriglohnsektors spielt hierbei eine besondere Rolle.

\section{Einleitung}

Die deutsche Arbeitsmarktpolitik der Nachkriegszeit lässt sich in vier wichtige Phasen einteilen: Erstens der Aufbau arbeitsmarktpolitischer Institutionen in der christdemokratischen Ära Adenauer/Erhard (1949-1966), zweitens die Etablierung aktiver Arbeitsmarktpolitik in der sozialliberalen Ära Brandt/Schmidt (1966-1982), drittens eine Arbeitsmarktpolitik nach Haushaltslage und die Ausweitung des arbeitsmarktpolitischen Instrumentariums zur sozialpolitisch motivierten Abfederung des massiven Wegbruchs von Arbeitsplätzen in Ostdeutschland in der christlichliberalen Ära Kohl (1982-1998) und schließlich viertens die Ära Schröder (1998-2005) mit ihrer umstrittenen „Agenda 2010“.

In die Endphase der christlich-liberalen Ära Kohl fällt die grundlegende Reform des AFG (1997), das die Arbeitsförderung in das Sozialgesetzbuch (SGB III) integrierte. Mit diesem von der FDP und dem wirtschaftsorientierten Flügel der CDU geprägten Gesetz wurde auf die bis dahin de lege geltenden Zielgrößen „hoher Beschäftigungsstand“ und „Vermeidung unterwertiger Beschäftigung" verzichtet und als neues Leitbild die Selbstverantwortung der Arbeitnehmerinnen und Arbeitnehmer formuliert (Schmid 2006, S. 192). Die liberale Ausrichtung der Ära Kohl spiegelt sich in Maßnahmen zum Sozialabbau und zur Deregulierung des Arbeitsrechts wider: So wurden die Regelungen über die Zumut- barkeit einer neuen Beschäftigung für Arbeitslose verschärft, Lohnersatzleistungen im Krankheitsfall vermindert, der Abschluss befristeter Arbeitsverträge erleichtert und der Geltungsbereich des Kündigungsschutzes eingegrenzt. Weiterhin sollten mit der Organisationsreform „Arbeitsamt 2000" die Dienstleistungen der Bundesanstalt für Arbeit grundlegend modernisiert werden.

Die rot-grüne Koalitionsregierung startete mit dem Versprechen, einige „Zumutungen “ der Ära Kohl wieder zurückzunehmen, was beispielsweise in den Bereichen Kündigungsschutz und Lohnfortzahlung im Krankheitsfall auch umgesetzt wurde. Ebenfalls in dieser Phase trat die Neuregelung zur sozialversicherungs- und steuerrechtlichen Behandlung der geringfügig Beschäftigten in Kraft (1.4.1999). Ziel war es, die Ausweitung dieser Beschäftigungsform einzudämmen und die Verdrängung regulär sozialversicherungspflichtiger Arbeitsplätze zu reduzieren.

Nach dem Rücktritt Oskar Lafontaines vom Amt des Bundesfinanzministers im März 1999 setzte schrittweise eine Kehrtwende in der Arbeitsmarkt- und Beschäftigungspolitik der rot-grünen Koalitionsregierung ein. Sie war in der Folge stark von der angebotsorientierten Europäischen Beschäftigungsstrategie geprägt (Schmid/Kull 2004). Mit dem berühmten Schröder-BlairMemorandum „Der Weg nach vorne für Europas Sozialdemokraten" wurde deutlich, dass der Blick zunehmend nach außen und auf liberale Beschäftigungsregime gerichtet wurde. Den Kernpunkt dieses Memorandums stellte eine „neue angebots- orientierte Arbeitsmarktpolitik der Linken“ dar, u. a. mit der Forderung: „Der Arbeitsmarkt braucht einen Sektor mit niedrigen Löhnen, um gering Qualifizierten Arbeitsplätze verfügbar zu machen" (Schröder/Blair 1999). Diese nachholende inhaltliche Ausgestaltung der wahltaktischen Orientierung auf die „Neue Mitte“ stieß parteiintern und öffentlich auf große, auch kritische Resonanz.

Weniger beachtet wurde das JobAQTIV-Gesetz, das bereits im Januar 2002 in Kraft trat. Anstoß hierfür gab insbeson-

Frank Oschmiansky, Dipl.-Pol., wissen-
schaftlicher Mitarbeiter am Wissenschafts-
zentrum Berlin für Sozialforschung (WZB).
Arbeitsschwerpunkte: Implementation und
Evaluierung der Arbeitsmarktpolitik,
Geschichte der Arbeitsmarktpolitik,
atypische Beschäftigung.
e-mail: fosch@wzb.eu
Andreas Mauer, Dipl.-Soz., wissenschaft-
licher Mitarbeiter am Wissenschaftszentrum
Berlin für Sozialforschung (WZB).
Arbeitsschwerpunkte: Arbeitsmarkt- und
Sozialpolitik, sozialwissenschaftliche
Statistik, mikroökonometrische Wirkungs-
analysen.
e-mail: mauer@wzb.eu.
Karin Schulze Buschoff, Dr. phil., wissen-
schaftliche Mitarbeiterin am Wissenschafts-
zentrum Berlin für Sozialforschung (WZB).
Arbeitsschwerpunkte: Arbeitsmarkt- und
Sozialpolitik im europäischen Vergleich.
e-mail: buschoff@wzb.eu


dere der Beginn der europäischen Beschäftigungspolitik durch den im November 1997 beschlossenen „Luxemburg-Prozess“. Das Job-AQTIV-Gesetz griff das in den europäischen beschäftigungspolitischen Leitlinien verankerte Ziel der Verbesserung der Beschäftigungsfähigkeit vor allem durch Profiling und Assessment, Jobrotation, Eingliederungsvereinbarung, Beschäftigung schaffende Infrastrukturförderung und Förderung besonderer Beschäftigtengruppen auf. Erst durch den sogenannten Vermittlungsskandal ${ }^{1}$ Anfang des Jahres 2002 trat die Arbeitsmarktpolitik wieder in den Vordergrund. Steigende Arbeitslosenzahlen und die Rezession verschärften zudem den Problemdruck.

Als Reaktion auf den Vermittlungsskandal wurde ein Expertengremium, die sogenannte Hartz-Kommission, damit beauftragt, Vorschläge zu unterbreiten, wie die Arbeitsmarktpolitik in Deutschland effizienter gestaltet und die Bundesanstalt für Arbeit reformiert werden könnte. Im August 2002 legte die Kommission ihre Vorschläge vor, die Schröder (so seine Ankündigung in seiner Regierungserklärung im Oktober 2002) „Eins zu Eins“ umsetzen wollte. Die Vorschläge der Kommission betrafen vor allem verschiedene Ansätze zur Beschleunigung der Arbeitsvermittlung, Personal-Service-Agenturen (PSA) zur Verbesserung der Vermittlungschancen von Arbeitslosen durch Arbeitnehmerverleih, sozialversicherungsfreie Minijobs für Dienstleistungen insbesondere in privaten Haushalten, niedrigschwellige Existenzgründungen durch dreijährige Zuschüsse (Ich-AG), die Reorganisation der Bundesanstalt für Arbeit zu einem modernen Dienstleistungsunternehmen (Bundesagentur für Arbeit) sowie die Zusammenlegung von Arbeitslosenhilfe und Sozialhilfe zu einer Grundsicherung für Langzeitarbeitslose und erwerbsfähige Sozialhilfeempfänger, dem sogenannten Arbeitslosengeld II. ${ }^{2}$

Wir wollen in diesem Beitrag zeigen, in welcher Weise die Reformen der letzten Jahre die generelle Stoßrichtung der deutschen Arbeitsmarktpolitik verändert haben. Die zentrale These lautet, dass eine Vielzahl kleinerer und größerer gesetzlicher Änderungen und damit einhergehende bzw. parallel dazu verlaufende Entwicklungen in der Vermittlungs- und Förderungspraxis in der Summe zu einem Paradigmenwechsel von der aktiven zu einer autoritär-aktivierenden Arbeitsmarktpoli- tik geführt haben. Eine zentrale Bedeutung kommt dabei der Förderung des Niedriglohnsektors zu. Bevor wir darauf in Abschnitt 3 ausführlicher eingehen, werden in Abschnitt 2 zunächst zentrale Entwicklungslinien der Arbeitsmarktpolitik der vergangenen Jahre nachgezeichnet. In Abschnitt 4 diskutieren wir in aller Kürze, warum die Niedriglohnstrategie in sozial-, aber auch arbeitsmarktpolitischer Hinsicht eine Sackgasse ist. Abschnitt 5 schließt mit einem Plädoyer für die Reaktivierung einer fördernden aktiven Arbeitsmarktpolitik, die durch die Überbetonung des Aspekts des Forderns und des Vorrangs einer möglichst schnellen Vermittlung in Beschäftigung - auch in prekäre und/oder nicht existenzsichernde Arbeitsverhältnisse - aus den Augen verloren wurde.

\section{Die Entwicklung der Arbeitsmarktpolitik in den letzten Jahren}

Die Einführung der „Grundsicherung für Arbeitssuchende“ (ALG II) im Jahr 2005 war zweifellos eine radikale Reform. Sie verlässt einen jahrzehntelangen Entwicklungspfad (Mohr 2004) und stellt einen tiefen Einschnitt in sozialstaatliche Traditionen dar. Für die ehemaligen Arbeitslosenhilfebezieher bedeutete dies, dass die nach dem Lohnprinzip bemessene Arbeitslosenhilfe abgeschafft und durch ein strikt nach Sozialhilfebedarf berechnetes und streng bedürftigkeitsgeprüftes Arbeitslosengeld II ersetzt wurde. Durch die Hartz-IV-Reform ist zudem die "unerschütterliche ,Pfadabhängigkeit‘ der institutionellen Finanzierungsstruktur" (Schmid/Oschmiansky 2007) aufgebrochen worden. Zum einen übernimmt nunmehr der Bund einen größeren Anteil an der Finanzierung der passiven Leistungen für Langzeitarbeitslose, zum anderen finanziert er jetzt auch die Eingliederungsleistungen für Langzeitarbeitslose und andere ALG-II-Empfänger, was 2006 immerhin ca. 4 Mrd. $€$ ausmachte. Diese Verschiebung der Finanzierungsstruktur ermöglichte letztendlich auch die stärkste Beitragssenkung in einem Sozialversicherungszweig in der Geschichte der Bundesrepublik Deutschland. ${ }^{3}$

Verbunden damit waren in den letzten Jahren diverse Leistungskürzungen in den Bereichen sowohl der aktiven als auch der passiven Arbeitsmarktpolitik, die zum Teil schon im Vorfeld der Hartz-Reformen stattgefunden hatten. So setzten bei den passiven Leistungen die Kürzungen für Langzeitarbeitslose bereits 2002 durch verschärfte Regeln der Anrechnung von Einkommen und Vermögen ein. ${ }^{4}$ Im Bereich des SGB III ist insbesondere die reduzierte Arbeitslosengeldbezugsdauer für Ältere zu nennen (Gesetz zu Reformen am Arbeitsmarkt), aber auch die Verkürzung der Rahmenfrist für die Anwartschaft auf Arbeitslosengeld (Drittes Gesetz für moderne Dienstleistungen am Arbeitsmarkt). ${ }^{5}$

Insgesamt kommt dem Förderaspekt in der Arbeitsmarktpolitik eine immer nachrangigere Bedeutung zu. Der Anteil der Ausgaben für aktive Arbeitsmarktpolitik an allen Ausgaben für Arbeitsmarktpolitik ist in den vergangenen drei Jahren auf den niedrigsten Stand seit Inkrafttreten des AFG im Jahr 1969 gesunken (Abbildung 1). Die Bundesagentur für Arbeit (BA) gab im Bereich des SGB III im Jahr 2006 nur ein Viertel der Beitragseinnahmen der Arbeitslosenversicherung für aktive Arbeitsfördermaßnahmen aus. Standen im Jahr 2000 noch knapp $4.500 €$ pro Leistungsempfänger im Eingliederungstitel zur Verfügung, so ist dieser Betrag in den letzten beiden Jahren auf unter $2.000 €$ gesunken. Im Be-

\footnotetext{
1 Im Januar 2002 monierte der Bundesrechnungshof Fehler in der Vermittlungsstatistik der damaligen Bundesanstalt für Arbeit. Weitere Überprüfungen ergaben, dass nur ein Drittel der ausgewiesenen Vermittlungen der gesetzlichen Definition entsprach, ein weiteres Drittel in einer interpretationsfähigen Grauzone lag und ein letztes Drittel nicht nachvollziehbar oder fingiert war.

2 Das Ergebnis langwieriger und schwieriger Verhandlungen bei allen vier Hartz-Gesetzgebungsverfahren war schließlich, dass die Vorschläge der Kommission am Ende zu etwa einem Drittel originalgetreu, zu einem Drittel mit starken Abweichungen und zu einem Drittel gar nicht umgesetzt wurden (Jann/Schmid 2004).

3 Zu den weiteren Gründen für den Rekordüberschuss der BA im Jahr 2006 vgl. Adamy (2007, S. 7).

4 Dies veranlasste den damaligen Finanzminister Hans Eichel zu der Feststellung: „Allein 2003 kürzen wir die Ausgaben für die Arbeitsmarktpolitik, die Bundesanstalt für Arbeit, die Arbeitslosenhilfe und die Rente um insgesamt 9,5 Mrd. $€$. Das ist eine riesige Kraftanstrengung. Vor allem bei der Arbeitslosenhilfe nehmen wir den Leuten richtig Geld weg." (Handelsblatt vom 21.11.2002)

5 Die Rahmenfrist (§ 124 SGB III) stellt den Zeitraum dar, innerhalb dessen die anwartschaftsbegründenden Zeiten (zwölf Monate; § 123 SGB III) zurückgelegt weren müssen, um eine Anwartschaft auf Arbeitslosengeld zu begründen.
} 


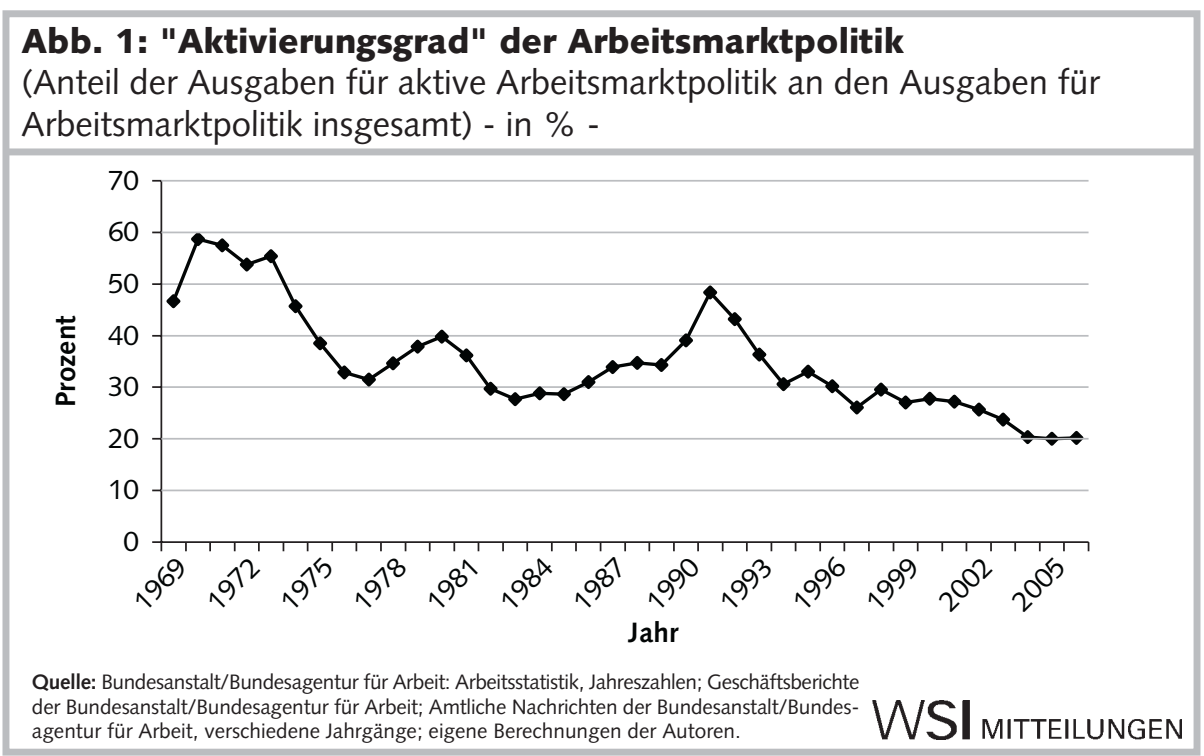

reich des SGB II ist die Bilanz noch ernüchternder: Nur 12,6 \% der Gesamtausgaben für das SGB II wurden im Jahr 2006 für aktive Maßnahmen verwendet. ${ }^{6}$ Der geringe Aktivierungsgrad der Arbeitsmarktpolitik steht im Widerspruch zu der Forderung, die stärkere Aktivierung der Arbeitsmarktpolitik mit der Umschichtung von Mitteln von der passiven zur aktiven Arbeitsmarktpolitik zu verbinden (Schmid 2002, S. 169f.).

\subsection{FÖRDERINSTRUMENTE}

Auch bei den Förderinstrumenten sind klare Akzentverschiebungen zu erkennen (Abbildung 2): Die Agenturen für Arbeit setzen vermehrt auf Trainingsmaßnahmen $^{7}$ statt auf Weiterbildungsmaßnahmen, nicht zuletzt weil Zeiten in Trainingsmaßnahmen anders als solche in Weiterbil-

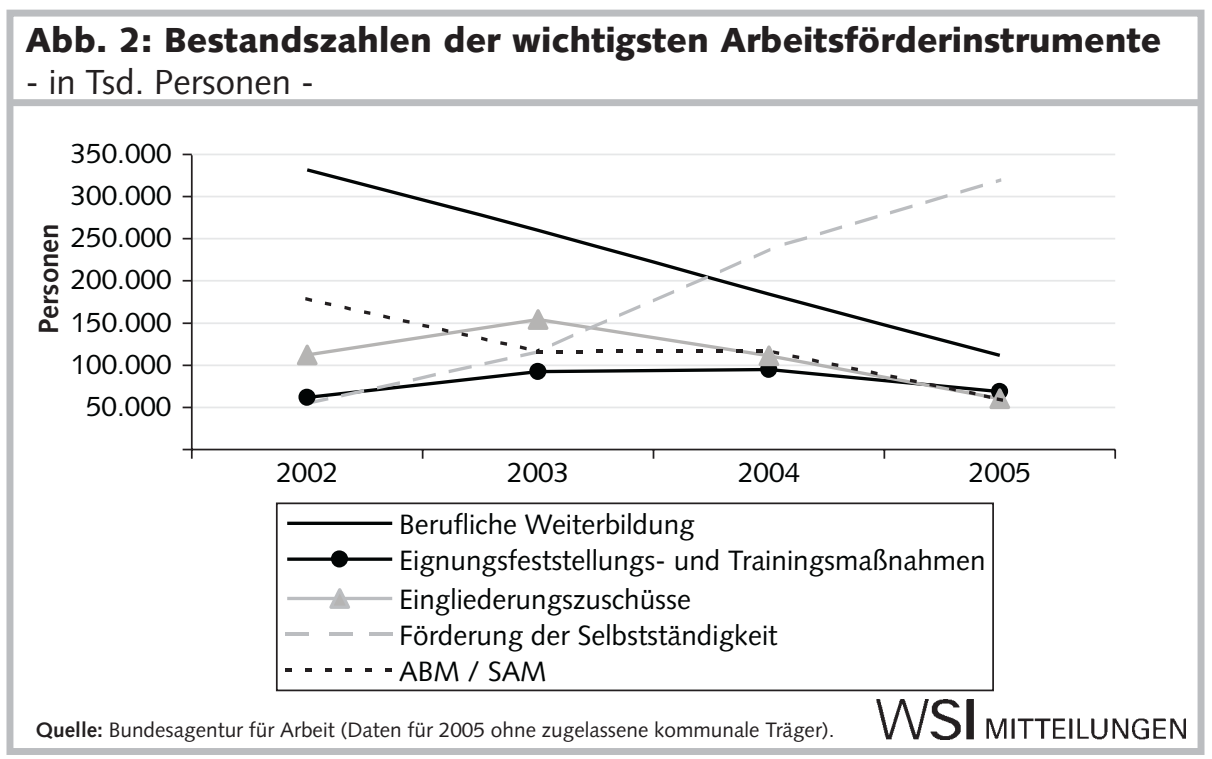

beitsgelegenheiten (Jahresdurchschnittsbestände) betrug hier im Jahr 2005 1:10. Weit weniger noch als im SGB III ist im SGB II ein ausgewogenes Verhältnis zwischen „Fordern“ und „Fördern“ zu erkennen. Gleich an prominenter Stelle $(\$ 2$, Grundsatz des Forderns) wird das wesentliche Element des "workfare-Ansatzes“ (keine Leistung ohne Gegenleistung) etabliert. Die geforderten Gegenleistungen gehen im SGB II weit über Etabliertes hinaus. Während Eigenaktivitäten umfassend abverlangt (deren Nichtbeachtung sanktioniert werden kann) und in einer Eingliederungsvereinbarung festgehalten werden (als rechtlich bindender öffentlich-rechtlicher Vertrag mit sanktionsbewehrter Teilnahmepflicht), stehen dem - jenseits der existenzsichernden Geldleistungen - keine gesicherten Rechtsansprüche auf passgenaue, ausreichende und erfolgversprechende Eingliederungsleistungen gegenüber.

Auch das mit dem Fortentwicklungsgesetz ins SGB II eingefügte Sofortangebot ( $\$ 15 a)$ ist weniger als Erweiterung des Förderspektrums, sondern vielmehr als Kontroll- und Sanktionsnorm konzipiert, was sich zum einen daran zeigt, dass im Sanktionsparagrafen das Sofortangebot ausdrücklich erwähnt wird, und zum anderen daran, dass der Gesetzgeber vom Sofortangebot Einsparungen in Höhe von 280 Mio. $€$ jährlich erwartet (BT-Drs. 16/1410, S. 36). Repressive Elemente im SGB II werden nicht zuletzt dadurch deutlich, dass ausgesprochene Sanktionen selbst dann aufrechterhalten bleiben, wenn der erwerbsfähige Hilfebedürftige sein Verhalten ändert ( $\$ 31$ SGB II).

Letztlich verstärkt das SGB II - insbesondere mit der Zumutbarkeitsregelung des $\S 10$ - die Tendenz, Arbeitslosigkeit über die verstärkte Deregulierung des Arbeitsmarktes verringern zu wollen, wie zuletzt beispielsweise durch Änderungen im Kündigungsschutzgesetz, die Befristung von Arbeitsverhältnissen und die Handwerksordnung. Damit einher geht der Rückgang des sogenannten Normalarbeitsverhältnisses, also der sozial abgesicherten abhängigen und unbefristeten Vollzeitbe-

6 Daten nur für ARGEn und getrennte Aufgabenwahrnehmung, ohne zugelassene kommunale Träger.

7 Die Bestandszahlen bei Trainingsmaßnahmen steigen nicht aufgrund der immer kürzeren Maßnahmedauer. 
schäftigung. Waren im Jahr 2001 noch $37,2 \%$ der Personen im erwerbsfähigen Alter in einem so definierten Normalarbeitsverhältnis tätig, so sank dieser Anteil innerhalb von nur vier Jahren auf 33,9 \% (Oschmiansky 2007).

\subsection{NEUE ZIELGRUPPEN}

Neben der Instrumentenverschiebung haben sich auch die Zielgruppen der Arbeitsmarktpolitik verschoben. War das Arbeitsförderungsrecht bis vor wenigen Jahren stark auf die Förderung von „besonders förderungsbedürftigen Personengruppen“ des Arbeitsmarktes ausgerichtet ( $\$ \$ 6$ und 7 SGB III, a. F.), so ist diese Zielsetzung mit dem Job-AQTIV-Gesetz aus den Einleitungsvorschriften des SGB III verschwunden. Jetzt wird nur noch auf die Verkürzung der Dauer der Arbeitslosigkeit $(\$ 1$ [1] SGB III) abgestellt. Die Einführung der Eingliederungsbilanzen und das mittlerweile etablierte Benchmarking verstärkten das Creaming, d.h. die selektive Förderung der „Besten“, bei arbeitsmarktpolitischen Maßnahmen zuungunsten des benachteiligten Klientels weiter. Die Handlungsprogramme (für Arbeitnehmerkunden) der BA verstärkten diesen Trend nochmals, indem Arbeitslose mit geringen Integrationschancen (Betreuungskunden) weitgehend von arbeitsmarktpolitischen Maßnahmen ausgeschlossen werden (ausführlicher WZB/infas 2006, S. 83 ff.). Dass zu der neuen sogenannten ,aktivierenden Arbeitsmarktpolitik" nicht mehr explizit die Stärkung der Chancengleichheit ausgegrenzter Gruppen zählt, stellt nicht nur einen Bruch mit der jahrzehntelangen Tradition der Arbeitsmarktpolitik in Deutschland dar. Es ist auch ein immanenter Bruch im Konzept selbst, zumal gezielte Maßnahmen zur Förderung der Beschäftigungsfähigkeit sehr gut geeignet sind, Arbeitslose zu ,aktivieren“, indem ihre Chancen auf dem Arbeitsmarkt erhöht werden (Bieback 2004, S. 82).

\section{Von der aktiven zur "Autoritär aktivierenden" Arbeitsmarktpolitik}

Zusammenfassend lässt sich festhalten, dass ein Politikwechsel von der aktiven hin zu einer ,autoritär aktivierenden“ Arbeits- marktpolitik stattgefunden hat. Einen prominenten Platz nimmt dabei die Verschärfung der Anspruchsvoraussetzungen für Transferleistungen nach der Maxime „Leistung und Gegenleistung “ ein. Wer die geforderte Gegenleistung nicht erbringt bzw. im Einzelfall nicht nachweisen kann, dass er sich regelkonform verhalten hat (Beweislastumkehr), wird mit dem (temporären) Entzug der Geldleistungen bestraft: So steht beispielsweise für Arbeitslosengeld-II-Bezieher, die sich nicht „einbringen" wollen oder können, ein umfangreiches Sanktionsinstrumentarium zur Verfügung, das bis zur Streichung sämtlicher Leistungen, einschließlich der Kosten der Unterkunft, führen kann. Anspruchsvolle und in der Regel teurere Instrumente der aktiven Arbeitsmarktpolitik wie Fortbildung und Umschulung, aber auch Arbeitsbeschaffungsmaßnahmen, werden zugunsten von kurzen Trainingsmaßnahmen, Eingliederungszuschüssen oder der Beauftragung von Dritten mit Vermittlungsdienstleistungen zurückgefahren. ${ }^{8}$ Arbeitslosen, die nur geringe Chancen am Arbeitsmarkt haben, werden zunehmend arbeitsmarktpolitische Instrumente zur Verbesserung ihrer Vermittlungschancen vorenthalten. Des Weiteren werden mit Ich-AGs, Mini- und Midijobs sowie Arbeitsgelegenheiten („Ein-Euro-Jobs“) Formen nicht existenzsichernder Beschäftigung in hohem Umfang gefördert.

Inwieweit aber lässt sich in diesem $\mathrm{Zu}$ sammenhang von einem Paradigmenwechsel sprechen? In Anlehnung an Peter Hall (1993) lassen sich politische Reformen als Prozesse beschreiben, die drei zentrale Punkte umschließen: erstens die damit verbundenen Ziele, zweitens die eingesetzten Instrumente und drittens die genaue Ausgestaltung dieser Instrumente. Je nachdem, ob bzw. inwieweit diese drei Größen berührt werden, lassen sich Reformen erster, zweiter und dritter Ordnung unterscheiden. Von einer Reform erster Ordnung spricht man, wenn lediglich Instrumente modifiziert werden (z. B. die Senkung des Unterhaltsgeldes bei Teilnahme an einer Fortbildungsmaßnahme durch das Erste Gesetz zur Umsetzung des Spar-, Konsolidierungs- und Wachstumsprogramms im Jahr 1994), das Instrument und auch das damit verbundene Ziel davon aber unberührt bleiben. Dagegen zeichnet sich eine Reform zweiter Ordnung dadurch aus, dass die Instrumente selbst verändert werden, die damit verbundenen Ziele je- doch dieselben bleiben (z. B. die Reform der beruflichen Weiterbildung durch das Erste Gesetz für moderne Dienstleistungen am Arbeitsmarkt mit der Umstellung auf ein Gutscheinsystem). Reformen dritter Ordnung schließlich gehen darüber hinaus mit einer veränderten Zielsetzung der Politik - einem Paradigmenwechsel - einher (Schmid 2003).

Betrachtet man die arbeitsmarktpolitischen Reformen der vergangenen Jahre, so findet man eine Vielzahl von Reformen erster und zweiter Ordnung, wobei der Politikwechsel nicht erst mit den HartzGesetzen, sondern schon vorher mit dem Job-AQTIV-Gesetz (2002) oder gar bereits mit dem Übergang vom AFG zum SGB III (1997) eingesetzt hat (z. B. die Abschaffung des temporären Qualifikationsschutzes in der Zumutbarkeitsregelung). In einzelnen Bereichen bewirkten die Reformen der letzten zehn Jahre die größten Akzentverschiebungen in der Arbeitsmarktpolitik seit der Einführung des AFG im Jahr 1969.

Im Bereich der aktiven Arbeitsmarktpolitik sind neben pfadabhängigen Reformen auch Veränderungen der Zielsetzungen zu verzeichnen. In der bisherigen Logik verbleibende pfadabhängige Krisenbewältigungsstrategien sind seit der ersten Krise auf dem Arbeitsmarkt 1975 die Verschärfung von Sanktionsregeln und Zumutbarkeitsanforderungen sowie die Verschiebung von Pflichtleistungen in den Bereich der Ermessensleistungen (letzte Pflichtleistung ist nunmehr der Anspruch auf Vermittlungsdienstleistungen durch Dritte nach $\S 37$ sowie $\$ 421 \mathrm{~g}$ ). Neu ist jedoch die Dynamik der Entwicklungen in den letzten Jahren. Neu ist ferner die Akzentverschiebung weg von traditionellen Maßnahmen wie ABM und FbW zugunsten arbeitsmarktpolitischer Förderung prekärer Beschäftigungsverhältnisse (PSA, Ich-AG, Mini-Jobs etc.) sowie die Umstellung der Beschaffung von aktiven Maßnahmen vom Zuwendungsrecht auf das Vergaberecht (WZB/infas 2005, S. 285ff.). Mit der Entwicklung der Umsteuerung geht auch eine Verkürzung von Maßnahmelaufzeiten, eine Einschränkung der

8 Wobei Letzteres auch aufgrund negativer Wirkungsergebnisse bereits dort wieder zurückgenommen wird, wo es sich nicht um Pflichtleistungen des SGB III handelt. VgI. dazu ausführlich Kaps/Schütz (2007) 
Zielgruppen ${ }^{9}$ und Beschränkung flankierender sozialer Angebote einher.

Die Reduzierung der Ausgaben für FbW und Umschulung, die Absenkung des Niveaus der steuerfinanzierten Transferleistungen für Arbeitslose, dem Arbeitslosengeld II (Alg II) auf den Regelsatz der Sozialhilfe und die Förderung von prekären Beschäftigungsverhältnissen gepaart mit immer strikter werdenden Zumutbarkeitskriterien führen in der Summe zu einen Paradigmenwechsel in einem zentralen Bereich der Arbeitsmarktpolitik: nämlich die Abkehr von dem Ziel, Arbeitslose vorrangig in eine sozial gesicherte Beschäftigung (gegebenenfalls über den Umweg FbW) zu vermitteln. Stattdessen gewinnt eine Strategie an Bedeutung, die den Abbau der Arbeitslosigkeit nicht nur durch eine beschleunigte Vermittlung in den ersten Arbeitsmarkt, sondern auch durch die Etablierung bzw. Ausweitung eines Niedriglohnsektors zu erreichen sucht. Dies betrifft insbesondere Langzeitarbeitslose und andere benachteiligte Arbeitsmarktgruppen. Der Segregation des Arbeitsmarktes in einen primären, sozial abgesicherten und einen sekundären, prekären JedermannsArbeitsmarkt (Lutz/Sengenberger 1988) wird immer deutlicher eine analog dazu segregierte Arbeitsmarktpolitik gegenübergestellt. Die chancenreichen Arbeitslosen werden dabei schnellstmöglich in den primären Arbeitsmarkt vermittelt, die weniger chancenreichen hingegen mit dem Instrumentarium einer autoritär aktivierenden Arbeitsmarktpolitik dazu gedrängt, entweder eine Maßnahme zu beginnen, mit der vor allem die Arbeitswilligkeit getestet wird (Trainingsmaßnahmen, Arbeitsgelegenheiten), oder eine Beschäftigung im sekundären Arbeitsmarkt bzw. Niedriglohnsektor aufzunehmen.

Damit wurde ein Richtungswechsel in der Arbeitsmarktpolitik eingeschlagen, der sich an der Standarderklärung für die andauernde Arbeitsmarktmisere in Deutschland orientiert: die mit wenigen Ausnahmen unisono geteilte Einschätzung, dass die im internationalen Vergleich hohe Arbeitslosigkeit in Deutschland, die mit einem sehr hohen Anteil Langzeitarbeitsloser einhergeht, zu einem erheblichen Teil auf Defizite bei der Beschäftigung im Bereich einfacher Tätigkeiten oder personennaher Dienstleistungen und damit auf zu wenige Jobs im Niedriglohnbereich zurückzuführen sei (vgl. exemplarisch Zimmermann 2003; Sachverständigenrat 2002;
Scharpf 2001). Zur Förderung des Niedriglohnsektors mussten auf Seiten des Arbeitsangebotes, um es mit den Begriffen des Missbrauchsdiskurses auszudrücken, „negative Beschäftigungsanreize" abgebaut und gering entlohnte Tätigkeiten , attraktiver" gemacht werden (IZA 2003), indem der finanzielle Druck auf die Betroffenen durch die Absenkung von Leistungen und die Verschärfung von Anspruchsvoraussetzungen verstärkt wurde. Die Einführung der Grundsicherung für Arbeitsuchende im Verbund mit strengeren Zumutbarkeitskriterien, denen durch teils drastische Sanktionsdrohungen Nachdruck verliehen wird, wirken in diese Richtung. Gleichzeitig wurden durch die Förderung von Miniund Midijobs auch nachfrageseitig neue Anreize für mehr Beschäftigung im Niedriglohnsektor gesetzt.

\section{4 \\ Sackgasse Niedriglohn- beschäftigung}

Von einem zu kleinen Niedriglohnsektor konnte indessen schon vor den Hartz-Reformen keine Rede sein. Vorliegende Studien zeigen, dass der Anteil der Niedriglohnbeschäftigung in den vergangenen Jahren deutlich und im internationalen Vergleich überproportional zugenommen hat (Kalina/Weinkopf 2006; OECD 2006, S. 175). Der Niedriglohnanteil bei sozialversicherungspflichtigen Vollzeitbeschäftigten lag einer Studie des IAB zufolge in Westdeutschland in der ersten Hälfte dieses Jahrzehnts bei gut $15 \%$ (Rhein et al. 2005). Eine Untersuchung des DIW, die alle Erwerbstätigen einbezieht, kommt für $\mathrm{Ge}$ samtdeutschland sogar auf einen Anteil von knapp einem Viertel aller Beschäftigten (23,4\% insgesamt; 20,3\% Westdeutschland, 38,6\% Ostdeutschland; vgl. Goebel et al. 2005). Das IAT wiederum berechnet für alle Beschäftigten im Jahr 2004 einen Anteil von $21 \%$ im Niedriglohnbereich, was hochgerechnet mindestens 6 Mio. Niedriglohnbeschäftigte ergibt (Kalina/Weinkopf 2006). ${ }^{10}$ Damit liegt der Anteil der im Niedriglohnsektor Beschäftigten über dem EU-Durchschnitt (Europäische Kommission 2004; OECD 2006).

Eine Arbeitsmarktpolitik, zu deren zentralen Zielen die Vermittlung in prekäre oder schlecht bezahlte Jobs gehört, ist aus sozialpolitischer Sicht nur dann akzepta- bel, wenn die Beschäftigung im Niedriglohnbereich transitorisch ist, ihr also eine Brückenfunktion beim Übergang von Arbeitslosigkeit in eine existenzsichernde Beschäftigung des ersten Arbeitsmarktes zukommt. Dann nämlich könnte sie ein wirksames Mittel zur Vermeidung oder Bekämpfung von Langzeitarbeitslosigkeit mit all ihren negativen Auswirkungen auf die künftigen Arbeitsmarktchancen von Betroffenen sein (Schmid 2004). Allerdings erweist sich in Deutschland der Übergang von einem Job im Niedriglohnbereich in eine besser bezahlte Beschäftigung bisher als besonders schwierig. Gemeinsam mit den USA, Großbritannien und Frankreich verzeichnet Deutschland die geringste Aufwärtsmobilität unter Niedriglohnbeschäftigten (OECD 2006).

Die mit den Hartz-Reformen eingeführten Maßnahmen und Instrumente konnten diese Situation bisher nicht positiv beeinflussen. So erwiesen sich die Minijobs nicht als erfolgversprechende Brücke in eine sozialversicherungspflichtige Beschäftigung; die Personal-Service-Agenturen waren in dieser Hinsicht sogar kontraproduktiv (WZB/infas 2006). Auch spricht derzeit wenig dafür, dass Arbeitsgelegenheiten („Ein-Euro-Jobs“) als Brücke in den ersten Arbeitsmarkt fungieren könnten (Kettner/Rebien 2007). ${ }^{11}$ Alles in allem kann nicht davon ausgegangen werden, dass Beschäftigungen in prekären oder schlecht bezahlten Jobs einen Prozess der Aufwärtsmobilität auslösen, der aus Arbeitslosigkeit in eine besser bezahlte und/ oder sozial abgesicherte Beschäftigung führt.

\footnotetext{
9 Die Arbeitsvermittler der BA sortieren die Arbeitslosen in vier Kundengruppen: Marktkunden, Beratungskunden aktivieren, Beratungskunden fördern und Betreuungskunden. Der Mitteleinsatz konzentriert sich auf die Beratungskunden (vgl. ausführlich Schütz/Oschmiansky 2006).

10 Die Niedriglohnschwelle ist bei den Studien des IAT und des IAB als Bruttomonatseinkommen unterhalb von zwei Dritteln des Medians und bei der Studie des DIW als Bruttostundenlohn unterhalb von zwei Dritteln des Medians definiert (Rhein et al. 2005; Goebel et al. 2005; Kalina/Weinkopf 2006).

11 Hinzu kommt, dass Ein-Euro-Jobs zunehmend reguläre Arbeitsplätze verdrängen. Bei einer anonymen Befragung von Arbeitgebern hatte fast jeder Zweite angegeben, Ein-Euro-Jobber als Krankheitsvertretung oder zum Überstundenabbau einzusetzen und damit gegen die gesetzlichen Regelungen verstoßen zu haben (Kettner/Rebien 2007)
} 
Die Große Koalition bleibt in dieser Hinsicht dennoch der Linie der Regierung Schröder treu. Sie hatte sich im Koalitionsvertrag vom November 2005 darauf verständigt, dass der Niedriglohnsektor einer Neuregelung bedarf. Als Ziel wurde formuliert, „Menschen mehr als bisher die Möglichkeit auch zur Beschäftigung mit niedrigem Einkommen an[zu]bieten" (Bundesregierung 2005, S. 25). Dies soll durch die Einführung eines Kombilohnmodells gelingen, mit dessen Hilfe die materiellen Anreize zur Aufnahme einer niedrig entlohnten Beschäftigung erhöht werden sollen.

In der Kombilohndebatte wird häufig unterschlagen, dass es in Deutschland bereits eine Vielzahl von (realen und in der Planung befindlichen) Varianten von Kombilohnmodellen gibt (Jaehrling/Weinkopf 2006). Nennenswerte Beschäftigungseffekte durch Kombilöhne konnten bisher jedoch nicht nachgewiesen werden (Kaltenborn 2004). Hinzu kommt, dass beträchtliche Mitnahmeeffekte insbesondere von Seiten der Arbeitgeber kaum zu verhindern sind. Kombilohnmodelle könnten die immer größer werdende Zahl von nichttarifgebundenen Arbeitgebern nämlich dazu verleiten, sehr niedrige Löhne zu zahlen, weil sie davon ausgehen können, dass der Staat die Ausfallbürgschaft in Form von Lohnkostenzuschüssen übernimmt. Unbefristete und unspezifische Kombilohnmodelle bergen daher unkalkulierbare Risiken im Hinblick auf Finanzierbarkeit und Lohngefüge (Dietz et al. 2006; Bofinger et al. 2006). Kombilöhne sollten als Instrumente der Arbeitsmarktpolitik daher - wenn überhaupt - nur befristet und zielgruppenorientiert eingesetzt werden. Idealerweise sollten sie mit Qualifizierungselementen kombiniert werden (Schmid 2004). Flankiert werden sollten Kombilohnmodelle, wie beispielsweise in Großbritannien praktiziert, unbedingt durch einen gesetzlichen Mindestlohn, um Mitnahmeeffekte bzw. Lohndumping im Rahmen zu halten. Als Allheilmittel zur Verbesserung der Arbeitsmarktlage in Deutschland sind Kombilöhne allerdings nicht geeignet (Dietz et al. 2006).

\section{$(2)$ \\ Schlussfolgerungen - Für eine Wiederbelebung der aktiven Arbeitsmarkt- politik}

Die Reorganisation der Vermittlungsprozesse durch die Bundesagentur für Arbeit ist überwiegend reformwirksam und hat grundsätzliche Weichen für eine stärkere Dienstleistungsorientierung und verbesserte Leistungsfähigkeit in der Vermittlung gestellt (Schütz/Oschmiansky 2006; WZB/ infas 2006). Gleichzeitig sind die Mittel für aktive Arbeitsmarktpolitik und dabei insbesondere für Qualifizierungsmaßnahmen in den letzten Jahren erheblich gekürzt worden, obwohl die Ergebnisse verschiedener Studien (OECD 2004; Fitzenberger/ Speckesser 2005; Lechner et al. 2005; Biewen et al.2006) auf eine hohe Wirksamkeit deuten und sich bereits in vielen Segmenten eine Fachkräftelücke zeigt. Das heißt: Auf der einen Seite konnte die BA für das vergangene Jahr 2006 einen Überschuss in einer noch nie ereichten Höhe von über $11 \mathrm{Mrd} . €$ verzeichnen und auf der anderen Seite offene Stellen nicht besetzen, da den Arbeitslosen geforderte Qualifikationen fehlen.

Teile dieser Mittel wieder für Qualifizierung und Weiterbildung - also für die Aufgaben, für die sie ursprünglich geplant waren - zu verwenden, liegt nahe. Damit würde der Erkenntnis der Notwendigkeit des ,lebenslangen Lernens" und der Erhaltung der Beschäftigungsfähigkeit, der „,employability“, Rechnung getragen. Diese Grundsätze sind nicht zuletzt als Ziele der EU-Beschäftigungs- und Sozialpolitik festgeschrieben. Bildung ist in unserer zunehmend wissensbasierten Gesellschaft der Schlüssel für die Verteilung von Lebenschancen. Auch die Erkenntnis, dass Bildungsanforderungen der heutigen Arbeitswelt nicht mit dem Abschluss von Schulund Ausbildungszeiten enden, ist ein Gemeinplatz. Zudem werden Chancen und Risiken auf dem Arbeitsmarkt auch weiter- hin von der Qualifikation bestimmt. Die sogenannte qualifikationsspezifische Arbeitslosigkeit trägt seit Jahren dieselben Vorzeichen: je höher die Qualifikation, desto geringer das Arbeitslosigkeitsrisiko (Reinberg/Hummel 2005).

Ins Zentrum muss die Frage rücken, nicht ob, sondern welche Weiterbildungsangebote notwendig sind, um nicht an Markterfordernissen vorbei zu qualifizieren. Um besser zu ermitteln, wo Qualifizierungsbedarfe bestehen, sollten auf betrieblicher Ebene vermehrt Bedarfsanalysen und Personalentwicklungsplanungen unternommen werden. Auch auf regionaler Ebene sind handlungsrelevante Qualifikationsbedarfsanalysen möglich (z. B. PROSPECT in Nordrhein-Westfalen). Um eine Qualität von Weiterbildungsangeboten zu sichern, ist es zudem erforderlich, Angebote und Träger regelmäßig zu evaluieren. Auf der Basis positiver Evaluationen sollte die Verstetigung von Mitteln garantiert sein, damit Weiterbildungsträger eine Grundlage haben, ihre Implementationsstrukturen vor Ort (wieder) aufzubauen.

Weiterbildung sollte verstärkt in modularisierter Form angeboten werden, um zum einen auf die Person zugeschnittene Fördermaßnahmen vorhalten zu können und zum anderen die Wahrscheinlichkeit der Passung auf zukünftige Arbeitsplatzanforderungen zu erhöhen. Wichtig wäre es, den Arbeitslosen größere Entscheidungsspielräume bei der Wahl der adäquaten Maßnahme einzuräumen und nicht, wie zurzeit durch die relativ strikten Handlungsprogramme praktiziert, diese Spielräume einzuengen. Nur mit einer aktiven Einbindung der Arbeitslosen in Entscheidungsprozesse kann das Leitbild des „Förderns und Forderns" greifen.

Bildungs- und Weiterbildungsförderung bleiben vor den skizzierten Hintergründen die vielversprechendsten Formen aktiver Arbeitsmarktpolitik. 
Adamy, W. (2007): Arbeitsmarktpolitik: Jahresbilanz 2006 - Perspektiven für 2007, in: Soziale Sicherheit 1, S. 5-14

Bieback, K.-J. (2004): Umbau der Arbeitsförderung nach den Vorschlägen der Hartz-Kommission - Analyse und Würdigung der Reformen, in: Schriftenreihe des Deutschen Sozialrechtsverbandes 52, S. 59-84 Biewen, M./Fitzenberger, B./Osikominu, A./Völter, R./Waller, M. (2006): Beschäftigungseffekte ausgewählter Maßnahmen der beruflichen Weiterbildung in Deutschland. Eine Bestandsaufnahme, in: Zeitschrift für Arbeitsmarktforschung 3-4, S. 365-390

Bofinger, P./Dietz, M./Genders, S./Walwei, U. (2006): Vorrang für das reguläre Arbeitsverhältnis: Ein Konzept für Existenz sichernde Beschäftigung im Niedriglohnbereich. Gutachten für das Sächsische Ministerium für Wirtschaft und Arbeit (SWMA)

Bundesregierung (2005): Gemeinsam für Deutschland - mit Mut und Menschlichkeit. Koalitionsvertrag zwischen CDU, CSU und SPD;

11.11.2005

Dietz, M./Koch, S./Walwei, U. (2006): Kombilohn: Ein Ansatz mit Haken und Ösen. IAB Kurzbericht 3, Nürnberg

Europäische Kommission (2004): Employment in Europe: Recent Trends and Prospects, Luxemburg

Hall, P. A. (1993): Policy, Paradigms, Social Learning, and the State:

The Case of Economic Policymaking in Britain, in: Comparative Politics 3, S. 275-296

Fitzenberger, B./Speckesser, S. (2005): Employment Effects of the Provision of Specific Professional Skills and Techniques in Germany. IAB Discussion Paper 21, Nürnberg

Goebel, J./Krause, P./Schupp, J. (2005): Mehr Armut durch steigende Arbeitslosigkeit, in: Wochenbericht des DIW Berlin 10, S. 175-183

IZA (2003): Den Reformaufbruch wagen. Aufruf von 300 Ökonomen an die politischen Entscheidungsträger, in: IZA compact, Juli/August, Bonn Jaehrling, K./Weinkopf, C. (2006): Kombilöhne in Deutschland - neue Wege, alte Pfade, Irrweg?, hrsg. von der Friedrich-Ebert-Stiftung, Bonn Jann, W./Schmid, G. (Hrsg.) (2004): Eins zu Eins? Eine Zwischenbilanz der Hartz-Reformen am Arbeitsmarkt, Berlin

Kalina, T./Weinkopf, C. (2006): Mindestens sechs Millionen Niedriglohnbeschäftigte in Deutschland: Welche Rolle spielen Teilzeitbeschäftigung und Minijobs? IAT-Report 03, Wuppertal

Kaltenborn, B. (2004): Kombilöhne: Stand und Perspektiven. Beiträge zur Wirtschaftsforschung und Politikberatung 22, Berlin

Kaps, P./Schütz, H. (2007): Privatisierung von Arbeitsvermittlungsdienstleistungen. Eine Bestandsaufnahme deutscher und internationaler Erfahrungen. WZB Discussion Paper SP I 2007-101, Wissenschaftszentrum Berlin für Sozialforschung, Berlin

Kettner, A./Rebien, M. (2007): Soziale Arbeitsgelegenheiten - Einsatz und Wirkungsweise aus betrieblicher und arbeitsmarktpolitischer Perspektive. IAB-Forschungsbericht 2, Nürnberg

Lechner, M./Miquel, R./Wunsch, C. (2005): Long-run Effects of Public Sector Sponsored Training in West Germany. IAB Discussion Paper 3, Nürnberg

Lutz, B./Sengenberger, W. (1988): Segmentationsanalyse und Beschäftigungspolitik, in: Bolte, K. M. (Hrsg.): Mensch, Arbeit und Betrieb. Beiträge zur Berufs- und Arbeitskräfteforschung, Weinheim, S. 273-286
Mohr, K. (2004): Pfadabhängige Restrukturierung oder Konvergenz? Reformen in der Arbeitslosenversicherung und der Sozialhilfe in Großbritannien und Deutschland, in: Zeitschrift für Sozialreform, 3, S. 283-311 OECD (2004-2006): OECD Employment Outlook, Paris

Oschmiansky, H. (2007): Der Wandel der Erwerbsformen und der Beitrag der Hartz-Reformen: Berlin und die Bundesrepublik Deutschland im Vergleich. WZB Discussion Paper SP I 2007-104, Wissenschaftszentrum Berlin für Sozialforschung, Berlin

Reinberg, A./Hummel, M. (2005): Vertrauter Befund: Höhere Bildung schützt auch in der Krise vor Arbeitslosigkeit. IAB-Kurzbericht 9, Nürnberg

Rhein, T./Gartner, H./Krug, G. (2005): Niedriglohnsektor: Aufstiegschancen für Geringverdiener verschlechtert. IAB-Kurzbericht 3, Nürnberg Sachverständigenrat (SVR) (2002): Sachverständigenrat zur Begutachtung der gesamtwirtschaftlichen Entwicklung, Jahresgutachten 2002/03: "Zwanzig Punkte für Beschäftigung und Wachstum", Berlin Scharpf, F. W. (2001): Employment and the Welfare State: A Continental Dilemma?, in: Ebbinghaus, B./Manow, P. (Hrsg.): Comparing Welfare Capitalism, London, pp. 270-283

Schmid, G. (2002): Wege in eine neue Vollbeschäftigung. Übergangsarbeitsmärkte und aktivierende Arbeitsmarktpolitik, Frankfurt/Main Schmid, G. (2003): Gestaltung des Wandels durch wissenschaftliche Beratung. Das Bündnis für Arbeit und die Hartz-Kommission, in: Ramge, S./Schmid, G. (Hrsg.): Management of Change in der Politik?, Münster Schmid, G. (2004): Soziales Risikomanagement durch Übergangsarbeitsmärkte. WZB Discussion Paper SP I 2004-110, Wissenschaftszentrum Berlin für Sozialforschung, Berlin

Schmid, G./Oschmiansky, F. (2007): Arbeitsmarktpolitik und Arbeitslosenversicherung, in: Bundesministerium für Arbeit und Soziales/Bundesarchiv (Hrsg.): Geschichte der Sozialpolitik in Deutschland seit 1945, Bd. 11: Bundesrepublik Deutschland 1989-1994. Sozialpolitik im Zeichen der Vereinigung (Hrsg. G. A. Ritter), Baden-Baden (im Erscheinen) Schröder, G./Blair, T. (1999): Der Weg nach vorne für Europas Sozialdemokraten. Ein Vorschlag von Gerhard Schröder und Tony Blair vom 8. Juni 1999, in: Blätter für deutsche und internationale Politik 7, S. 887896

Schütz, H./Oschmiansky, F. (2006): Arbeitsamt war gestern. Neuausrichtung der Vermittlungsprozesse in der Bundesagentur für Arbeit nach den Hartz-Gesetzen, in: Zeitschrift für Sozialreform 1, S. 5-28

WZB/infas (2005): Evaluation der Maßnahmen zur Umsetzung der Vorschläge der Hartz-Kommission. Modul 1a: Neuausrichtung der Vermittlungsprozesse. Bericht 2005 für das Bundesministerium für Arbeit und Soziales, Bonn/Berlin

WZB/infas (2006): Evaluation der Maßnahmen zur Umsetzung der Vorschläge der Hartz-Kommission. Modul 1a: Neuausrichtung der Vermittlungsprozesse. Bericht 2006 für das Bundesministerium für Arbeit und Soziales, Bonn/Berlin (http://www.wz-berlin.de/ars/ab/ hartzevaluation.de.htm)

Zimmermann, K. F. (2003): Beschäftigungspotentiale im Niedriglohnsektor, in: DIW-Vierteljahrsheft zur Wirtschaftsforschung 1, S. 11-24 